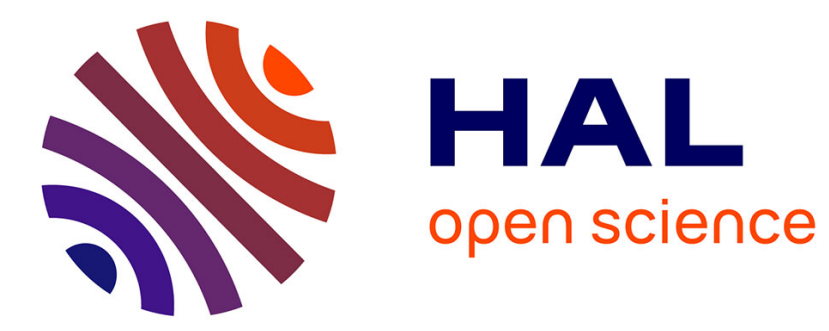

\title{
Experimental evaluation of obstacle clearance by a hybrid wheel- legged robot
}

Christophe Grand, Pierre Jarrault, Faïz Ben Amar, Philippe Bidaud

\section{To cite this version:}

Christophe Grand, Pierre Jarrault, Faïz Ben Amar, Philippe Bidaud. Experimental evaluation of obstacle clearance by a hybrid wheel- legged robot. 14th International Symposium on Experimental Robotics, 2014, Marrakech, Morocco. hal-03177935

\section{HAL Id: hal-03177935 \\ https://hal.science/hal-03177935}

Submitted on 23 Mar 2021

HAL is a multi-disciplinary open access archive for the deposit and dissemination of scientific research documents, whether they are published or not. The documents may come from teaching and research institutions in France or abroad, or from public or private research centers.
L'archive ouverte pluridisciplinaire HAL, est destinée au dépôt et à la diffusion de documents scientifiques de niveau recherche, publiés ou non, émanant des établissements d'enseignement et de recherche français ou étrangers, des laboratoires publics ou privés. 


\title{
Experimental evaluation of obstacle clearance by a hybrid wheel-legged robot
}

\author{
Christophe Grand, Pierre Jarrault, Faiz BenAmar, and Philippe Bidaud \\ ISIR (UMR-7222) UPMC/CNRS, Paris, France \\ christophe.grand@upmc.fr \\ http://www.isir.upmc.fr
}

\begin{abstract}
This paper deals with the problem of frontal obstacle crossing by a poly-articulated wheeled robot. We focus on the particular architecture of hybrid wheel-legged robots that are redundantly actuated systems. In this paper, experimental results that show the climbing capabilities of such system when crossing large obstacle are presented. We focus on the case of a step like obstacle whose height is superior to the diameter of the wheels. In this case, the adhesion properties have a large impact on the crossing capabilities. First, the control methodology based on both the optimization of the system posture and the distribution of internal forces is introduced. The optimization criterion represents the maximum allowable force disturbance that the system can support before violating the frictional contact constraint. Then, our experimental prototype, the robot Hylos2 and the experimental setup are presented. As our approach is based on the control of the contact forces, experiments used to quantify the level of friction in the mechanical transmissions are first reported. Then a step-crossing trial on the real system is presented.
\end{abstract}

Keywords: mobile robot, wheel-legged, high mobility

\section{Introduction}

In this paper, we address the motion control of poly-articulated mobile robots during obstacle clearance. We are focusing on a special class of mobile systems, often called hybrid wheel-legged robots, which are designed in order to increase both obstacle crossing and terrain adaptation capabilities.

The kinematic architecture of hybrid wheel-legged systems we consider in this work, is made of wheels mounted at the end of actuated legs. Many robots have already been developed based on this kinematic. Most of them are based on a 4 wheel-legs arrangement such as the Hylos robot [4, the Workpartner [1]. or the PAW robot [9. Some concepts use only three wheels, like the family of robots Tri-Star dedicated to planetary exploration [1], or the robot ROAMeR [3 that uses three active limbs with a steerable wheel at the end to form a planar reconfigurable omnidirectional mobile robot. Some few other systems use more than four legs like both the Athlete, developed by NASA/JPL [10, and the Asterisk-H, from the Arai Laboratory [12] which are using 6 wheel-legs. 
The control method developed in this paper is dedicated to the specific case of 4 wheel-legs robots and is validated on our experimental prototype Hylos2 (see Fig. 1). Its mechanical architecture is composed of 4 wheel-legs, each wheelleg being a multi-dof serial chain ended by a driven and steerable wheel. The nominal mechanical parameters are given in table 1 . This robot have the ability to change the position of its center of mass (CoM) and to modify the distribution of its contact forces. Furthermore, it is redundantly actuated systems exhibiting internal forces that should be optimized. The proposed motion controller is based on a torque control at the joints level that addresses the combined optimization of the internal forces and the CoM position, in order to maximize the contact stability (increasing traction and avoiding tip-over).

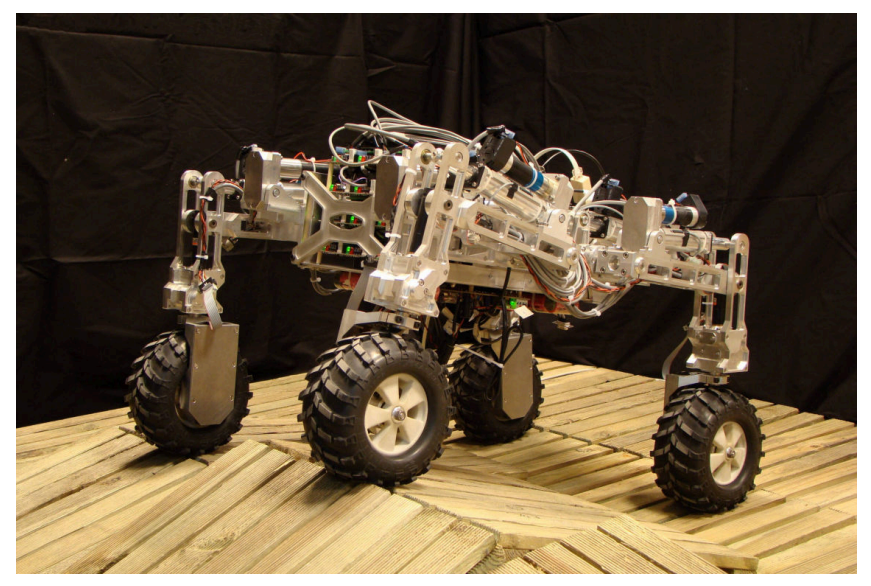

Fig. 1. Hylos2 prototype

\begin{tabular}{||ll||}
\hline Mass & $20 \mathrm{~kg}$ \\
Nominal velocity & $0.6 \mathrm{~m} / \mathrm{s}$ \\
Wheels diameter & $150 \mathrm{~mm}$ \\
Ground clearance & $100-300 \mathrm{~mm}$ \\
Nominal length & $700 \mathrm{~mm}$ \\
Width & $450 \mathrm{~mm}$ \\
\hline
\end{tabular}

Table 1. Nominal parameters of the Hylos2 robot

\section{Approach}

\section{$2.1 \quad$ System modeling}

We consider the general case of a system supported by $n$ wheel-legs (Fig. 2). The frame $\mathcal{R}_{p}=\left(G, \boldsymbol{x}_{p}, \boldsymbol{y}_{p}, \boldsymbol{z}_{p}\right)$ is attached to the main body of the system allowing 
to describe its motion with respect to the ground reference frame $\mathcal{R}_{0}$, and $G$ is the center of mass of the main body.

The $i^{\text {th }}$ leg is in a frictional contact with the ground at point $P_{i}$ which coordinates $\boldsymbol{p}_{i}$ are expressed in the frame $\mathcal{R}_{p}$ (Fig. 2). The contact force of the ground on each leg is denoted $\boldsymbol{f}_{i}=\left[f_{x_{i}} f_{y_{i}} f_{z_{i}}\right]^{t}$ where $f_{x_{i}}, f_{y_{i}}$ and $f_{z_{i}}$ are the components of the force along the contact frame's axis $\mathcal{R}_{c_{i}}=\left(P_{i}, \boldsymbol{x}_{i}, \boldsymbol{y}_{i}, \boldsymbol{z}_{i}\right)$, such that $\boldsymbol{z}_{i}$ is the contact normal and $\boldsymbol{x}_{i}, \boldsymbol{y}_{i}$ are the tangential directions.

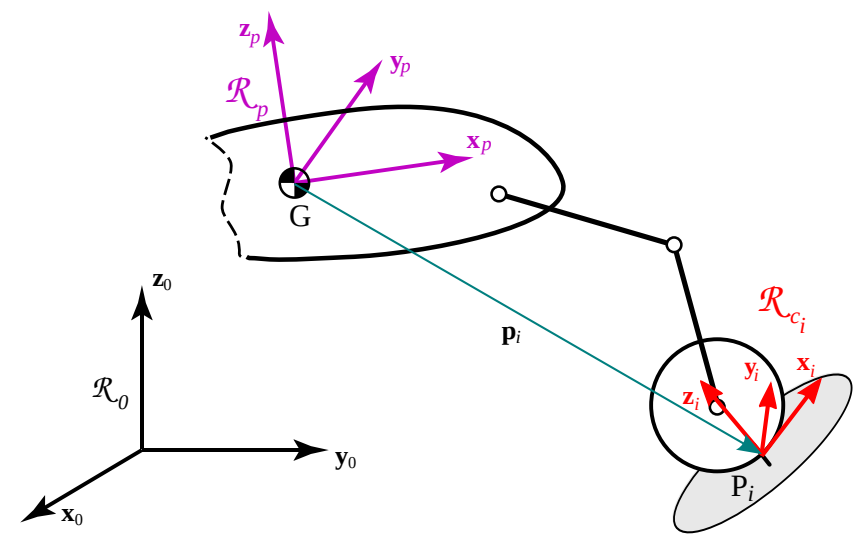

Fig. 2. Description of the $\mathrm{i}^{\text {th }}$ leg parameters

The equations describing the equilibrium of the system are given by:

$$
\mathrm{G} f=\boldsymbol{F}
$$

where $\boldsymbol{f}^{t}=\left[\boldsymbol{f}_{1}{ }^{t} \ldots \boldsymbol{f}_{n}{ }^{t}\right]$ is a $[3 n \times 1]$ vector containing all contact forces, expressed in each contact frame $\mathcal{R}_{c_{i}}$, and $\boldsymbol{F}$ is the set of external and inertial wrench, expressed in the local frame $\mathcal{R}_{p}$, that are applied to the platform at point $G$. $\mathbf{G}$ is a $[6 \times 3 n]$ matrix giving the equivalent wrench to the contact forces at the center of mass in the frame $\mathcal{R}_{p}$ (see [5] for more details):

$$
\mathbf{G}=\left[\begin{array}{ccc}
\mathbf{R}_{c_{1}}^{P} & \ldots & \mathbf{R}_{c_{n}}^{P} \\
\widetilde{\mathbf{p}}_{1} \mathbf{R}_{c_{1}}^{P} & \ldots & \widetilde{\mathbf{p}}_{n} \mathbf{R}_{c_{n}}^{P}
\end{array}\right]
$$

where $\mathbf{R}_{c_{i}}^{P}$ is the rotation matrix of the $\mathrm{i}^{\text {th }}$ contact frame $\mathcal{R}_{c_{i}}$ with respect to the platform $\mathcal{R}_{p}$, and $\widetilde{\mathbf{p}}_{i}$ is the skew-symmetric matrix of the cross product operator.

The contact forces must respect constraints related to actuators saturation and Coulomb friction law. The actuators limits are defined as follow:

$$
\left\{\begin{array}{r}
\mathbf{J}^{T} \boldsymbol{f}<\boldsymbol{\tau}_{\max } \\
-\mathbf{J}^{T} \boldsymbol{f}<\boldsymbol{\tau}_{\text {max }}
\end{array}\right.
$$


where $\mathbf{J}=\operatorname{blockdiag}\left(\mathbf{J}_{i}\right), \mathbf{J}_{i}$ being the Jacobian matrix of the $i^{\text {th }}$ leg and $\boldsymbol{\tau}_{\max }$ is the vector of the actuators torque limits.

The contact constraints are defined by Coulomb's friction law as a function of the contact forces:

$$
\left\{\begin{aligned}
\mu_{i} f_{z_{i}}^{2} & <f_{x_{i}}^{2}+f_{y_{i}}^{2} \\
f_{z_{i}} & >0
\end{aligned}\right.
$$

where $\mu_{i}$ is static friction coefficient at the contact. These constraints can be advantageously represented using a conservative pyramidal form adapted form [7] and given by:

$$
\mathbf{A}_{i} \boldsymbol{f}_{i}<0
$$

where

$$
\mathbf{A}_{i}=\left[\begin{array}{ccc}
0 & 1 & \frac{\mu_{i}}{\sqrt{2}} \\
0 & -1 & \frac{\mu_{i}}{\sqrt{2}} \\
1 & 0 & \frac{\mu_{i}}{\sqrt{2}} \\
-1 & 0 & \frac{\mu_{i}}{\sqrt{2}}
\end{array}\right]
$$

Thus we propose in this paper a stability criterion based on the smallest perturbation allowed at the contact level. This leads to to maximize robustness with respect to modeling errors affecting the contact force control. Let us define vector $\boldsymbol{d}$ as:

$$
\boldsymbol{d}=\mathbf{A} f
$$

where $\mathbf{A}$ is a matrix defined by $\mathbf{A}=\operatorname{blockdiag}\left(\mathbf{A}_{i}\right)$.

\subsection{Optimization procedure}

Considering the elements given in the previous section, the forces distribution problem is formulated as a "minimax" optimization procedure. Indeed, the objective is to maximize the smallest acceptable perturbation $(\phi=\min (\mathbf{A} \boldsymbol{f}))$ subject to the constraints (1) and (3):

$$
\begin{aligned}
\max _{\boldsymbol{f} \in \mathbb{R}^{3 n}} & \min (\mathbf{A} \boldsymbol{f}) \\
\text { s.t. } & \left\{\begin{array}{r}
\mathbf{G} \boldsymbol{f}=\boldsymbol{F} \\
\mathbf{J}^{T} \boldsymbol{f}<\boldsymbol{\tau}_{\max } \\
-\mathbf{J}^{T} \boldsymbol{f}<\boldsymbol{\tau}_{\max }
\end{array}\right.
\end{aligned}
$$

This problem can be transformed into its compact primal form ([2]). The solution $f$ of the optimization (7) should satisfy the force equilibrium equation constraint (1). This solution is divided in a particular solution $f_{p}$ and the homogeneous solution $\boldsymbol{f}_{h}$ :

$$
f=f_{p}+f_{h}
$$

The particular solution is chosen arbitrarily as the solution given by the weighted pseudo-inverse. The homogeneous solution, that correspond to the internal forces, is belong the null space of the matrix $\mathbf{G}$ expressing the force equilibrium equation. Let us denote $\left\{\boldsymbol{g}_{i}\right\}_{i \in[1, m]}$ the vectors defining a basis of the 


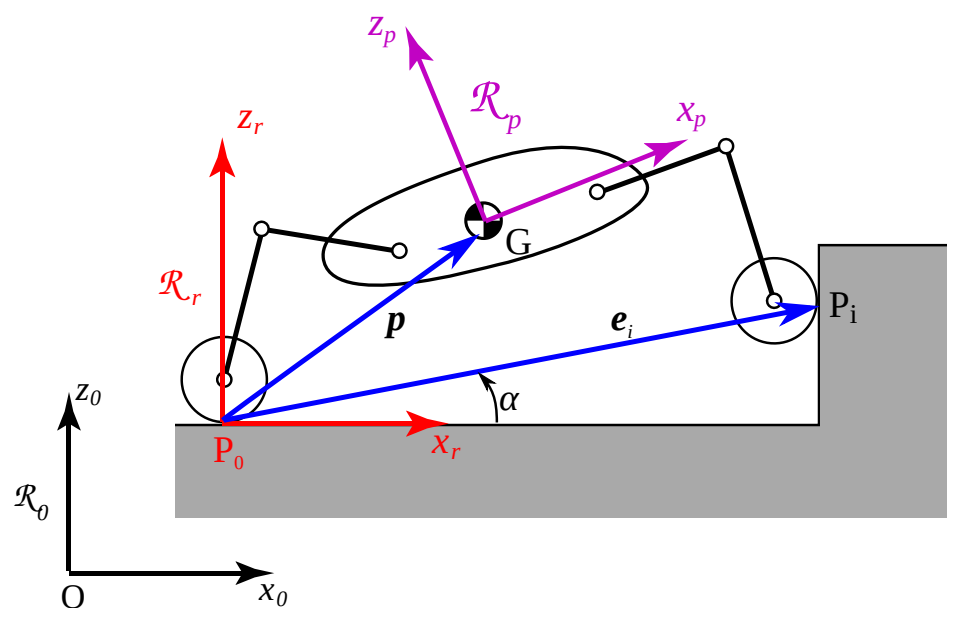

Fig. 3. Local frame and posture definition during frontal obstacle crossing

null space of $\mathbf{G}$, with $m=\operatorname{dim}(\mathbf{G})-\operatorname{rank}(\mathbf{G})$. The particular and homogeneous solutions can then be written as:

$$
\begin{aligned}
& \boldsymbol{f}_{p}=\mathbf{G}_{\text {norm }}^{+} \boldsymbol{F} \\
& \boldsymbol{f}_{h}=\mathbf{N}_{g} \boldsymbol{x}_{h}
\end{aligned}
$$

where $\mathbf{N}_{g}=\left[\boldsymbol{g}_{1} \ldots \boldsymbol{g}_{m}\right]=\operatorname{ker}(\mathbf{G})$.

This new formulation allows us to rewrite the optimization problem (7) by implicitly including the force equilibrium constraint, and to reduce the dimension of the optimization search space $(m<3 n)$ :

$$
\begin{aligned}
\max _{\boldsymbol{x}_{f} \in \mathbb{R}^{m}} & \min \left(\mathbf{A}\left[\boldsymbol{f}_{p}+\mathbf{N}_{g} \boldsymbol{x}_{f}\right]\right) \\
\text { s.t. } & \left\{\begin{array}{r}
\left(\mathbf{J}^{T} \mathbf{N}_{g}\right) \boldsymbol{x}_{f}<\boldsymbol{\tau}_{\max }-\mathbf{J}^{T} \boldsymbol{f}_{p} \\
-\left(\mathbf{J}^{T} \mathbf{N}_{g}\right) \boldsymbol{x}_{f}<\boldsymbol{\tau}_{\max }+\mathbf{J}^{T} \boldsymbol{f}_{p}
\end{array}\right.
\end{aligned}
$$

We consider in this paper the special case of a frontal obstacle crossing, as depicted in figure 3 , where the motion along the lateral direction has a negligible effect on the robot stability. Thus, we only consider the reconfiguration of the posture in the sagittal plane $\left(G, \boldsymbol{z}_{p}, \boldsymbol{x}_{p}\right)$. For these reasons and for sake of simplicity, we consider the motion of left and right sides of the robot as symmetrical.

Thus, we define $\boldsymbol{p}=\left[\begin{array}{ll}X & Z\end{array}\right]^{T}$ as the vector containing the longitudinal and vertical coordinates of the CoM position relatively to the rear wheel-soil contact point. To this purpose, an intermediate reference frame $\mathcal{R}_{r}$ aligned with the inertial frame $\mathcal{R}_{0}$ but attached to this point is defined.

In this case, the null space of $\mathbf{G}$ is entirely defined by the contact geometry (the angle $\alpha$ in the schema). For a fixed distance between contact points (the wheelbase $e_{i}$ ), the null space of $\mathbf{G}$ is not modified by a change of $X$ or/and $Z$. 
For a frontal crossing, i.e. the rotation between the ground and the contact's frame of the obstacle is around the lateral axis of the robot $\left(\boldsymbol{y}_{p}\right)$, the particular solution of force equilibrium equation can be defined as a combination of the CoM position $(X, Z)$ and the configruation of the contact angles (represented by the particular solution $f_{0}$ ):

$$
\boldsymbol{f}_{p}=\mathbf{G}_{\text {norm }}^{+} \boldsymbol{F}=\boldsymbol{c}_{x} X+\boldsymbol{c}_{z} Z+\boldsymbol{f}_{0}
$$

leading to the following general solution of the force equilibrium constraint:

$$
\boldsymbol{f}=\mathrm{N} \boldsymbol{x}+\boldsymbol{f}_{0}
$$

where

$$
\left\{\begin{aligned}
\mathbf{N} & =\left[\begin{array}{lll}
\mathbf{N}_{g} & \boldsymbol{c}_{x} & \boldsymbol{c}_{z}
\end{array}\right] \\
\boldsymbol{x}^{T} & =\left[\begin{array}{ll}
\boldsymbol{x}_{f}^{T} & \boldsymbol{p}^{T}
\end{array}\right]
\end{aligned}\right.
$$

Thus, the CoM position $\boldsymbol{p}$ is added to the vector of optimization variables and the combined optimization of both the internal forces and the posture can be formulated from (9) as:

$$
\begin{aligned}
\max _{\boldsymbol{x} \in \mathbb{R}^{m+2}} & \min \left(\overline{\mathbf{A}} \boldsymbol{x}+\overline{\boldsymbol{f}_{0}}\right) \\
\text { s.t. } & \left\{\begin{array}{r}
\overline{\mathbf{J}}^{T} \boldsymbol{x}<\boldsymbol{\tau}_{\max }-\mathbf{J}^{T} \boldsymbol{f}_{0} \\
-\overline{\mathbf{J}}^{T} \boldsymbol{x}<\boldsymbol{\tau}_{\max }+\mathbf{J}^{T} f_{0}
\end{array}\right.
\end{aligned}
$$

where

$$
\left\{\begin{array}{l}
\overline{\mathbf{A}}=\mathbf{A} \mathbf{N} \\
\overline{\mathbf{J}}^{T}=\mathbf{N}^{T} \mathbf{J} \\
\overline{\boldsymbol{f}_{0}}=\mathbf{A} \boldsymbol{f}_{0}
\end{array}\right.
$$

This optimization algorithm computes at each time step the optimal posture (position of the $\mathrm{CoM}$ ) and the optimal distribution of internal forces to be applied. The control of the robot posture requires the application of a position or velocity input at the joints level, whereas the control of internal forces needs a torque control. Thus, we have to face up with the problem of dual force/position control. In the present work, we propose to implement our controller by following the classical impedance control approach [6]. Indeed, the results obtained from the optimization do not necessarily involve that desired motion and force at the wheel contact will be orthogonal. Further, the possible incertitudes in the application of force, due to non-modelized friction in the mechanical transmissions, have a smaller impact on the quality of the impedance based control, compared to the hybrid force/position control.

The actuation torques at joint level are computed following this classical impedance control law:

$$
\boldsymbol{\tau}=\mathbf{K}_{q}\left(\dot{\boldsymbol{q}}^{d}-\dot{\boldsymbol{q}}\right)+\mathbf{J}^{T} \boldsymbol{f}
$$

where $\dot{\boldsymbol{q}}$ are the desired joint velocities and $\boldsymbol{f}$ is the force to be applied at the wheel-soil contacts. The contact forces are obtained from the solution $\boldsymbol{x}^{*}$ of the optimization (12) and the equation (11). Whilst the desired joint velocities $\dot{\boldsymbol{q}}$ are computed with the robot kinematic model, in order to maintain the posture to the desired value. More details concerning the posture control can be find in [5]. 


\section{Experimental results}

The experimental setup consists in an obstacle made with wooden planks, with a first inclined plane and a second horizontal plane. The angle of the first plan with respect to the horizontal ground is $60 \mathrm{deg}$ and its height is 19 centimeter. The friction coefficient between the wheels of the robot and the wood used for this setup has been evaluated experimentally. The estimated value is around $\mu \approx 0.8$.

The resulting obstacle represents a high difficulty with respect to the robot geometry, we indeed observed during our experiments that the joint positions were close to the mechanical limits. The evolution of the robot is represented with some snapshots of our experiments on the Fig. 6.

The global control law, including optimization loop, is implemented on the robot. The robot uses DC motors for each actuated joint (16 dof). The motors torque is controlled using a digital motion controllers from AMC company (Advanced Motion Control) that implement current loop control. The motor controllers are connected to an embedded computer through a CAN bus running at 1MBits/S and implementing the CANopen protocol. The PC-104 embedded computer is based on a Intel Atom N270 $(1.6 \mathrm{GHz})$ processor. The robot includes encoders at each joint to measure the kinematic parameters and an IMU (XSens MTi) to evaluate the attitude of the platform. The embedded computer is running with a real-time Xenomai/Linux kernel and the control softwares are deployed using the ROS middleware [8. The control is divided in two nodes: the first one runs as a $100 \mathrm{~Hz}$ real-time task to manage the low-level motion controllers distributed on the CAN bus and to compute the impedance control law (13); whilst the second one runs as non-RT task at $20 \mathrm{~Hz}$ to solve the optimization problem $(12)$ and send target position of the CoM and the desired interation forces to the previous node.

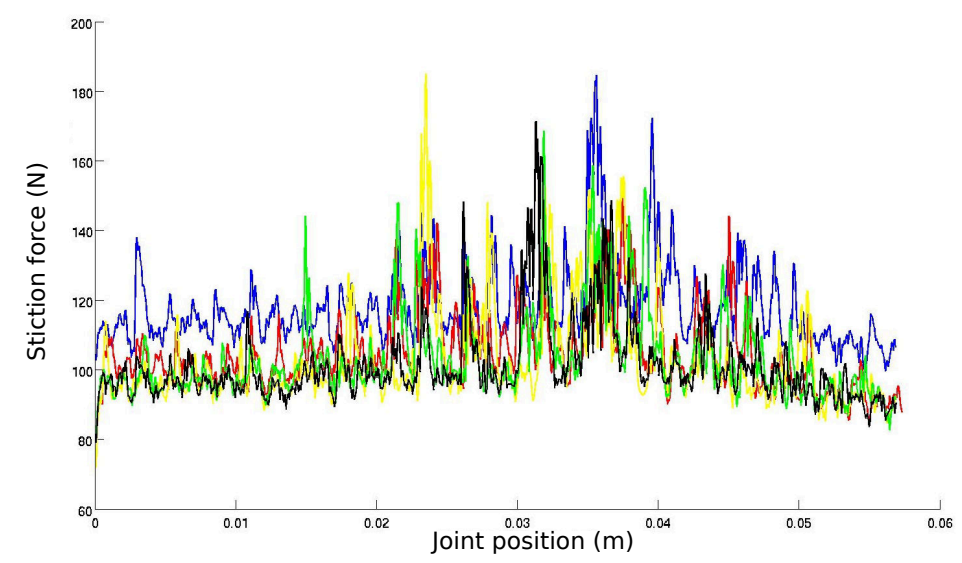

Fig. 4. Static friction 
One should note that this experimental prototype is not equipped with force sensors in the legs actuation chain. Thus, the required torque at joint level is directly obtained by controlling the current in the motors. Accordingly, the corresponding force that the robot is applying at the wheel-ground contact is not precisely known. Indeed, frictions in the mechanical transmission could affect these forces value. However, the choice of the stability margin used as criterion in the optimization procedure should be able to consider this effect as a perturbation, if we can check that the amount of friction force is less than the measured stability margin. To quantify the amount of friction force in the actuation system, we have applied a classical measurement protocol that consists to actuate each joint with out external contact force (no load motion) and to measure the resulting current in the motor. We have done two experiments: the first was to measure the needed torque to start the motion and that correspond to static friction (also called stiction force); the second was to measure the motor torque during the motion as a function of its position. The measurements are done during multiple trials on each actuators.

The figure 4 shows the results corresponding to the first type of tests (stiction value) that was obtained for one actuator whereas the figure 5 gives the results for the second type of tests (dynamic friction). As expected, we find that the stiction force is greater than the dynamic friction. Considering the geometry of the leg described by the jacobian matrix, the corresponding force at the wheelground contact point is, in the worst case, equal to $12 \mathrm{~N}$.

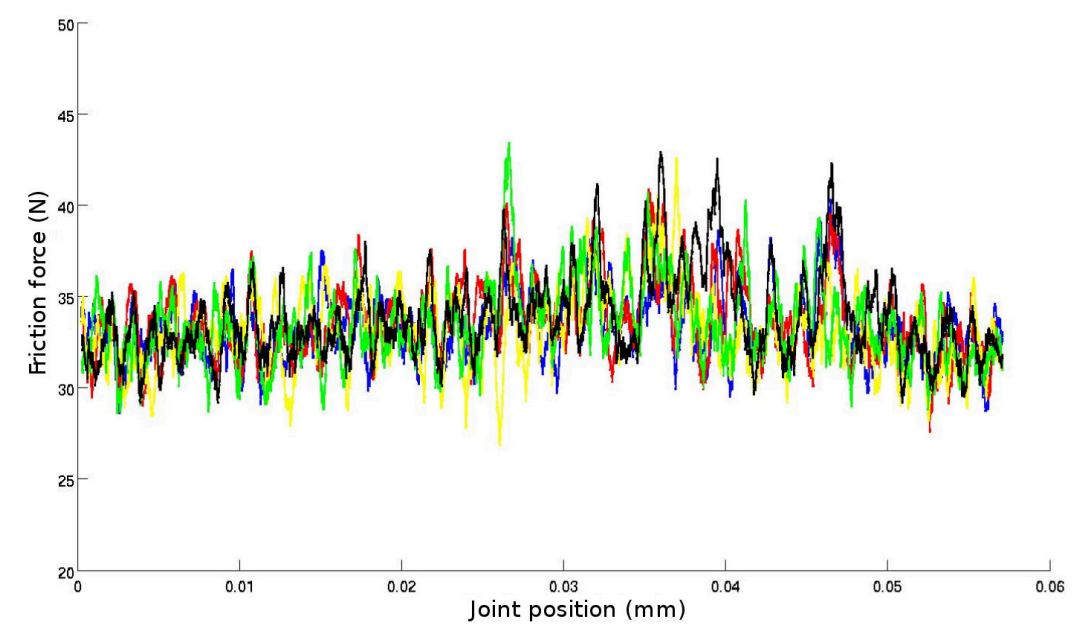

Fig. 5. Dynamic friction

The first result is that the robot is able to safely cross over a high step-like obstacle, despite the presence of important friction in the mechanical transmission 
that alter the precision of the impedance control. The Fig. 7 shows the obtained stability margin which represent the minimum residual tangential force of all the contact forces. This margin quantifies the distance to the unstable configuration arising from slippage. The minimum value is around $15 N$ that represents $10 \%$ of the system weight. This margin seems to be large enough but it should be compared to the internal friction force. Unfortunately, this value is not measurable directly on the robot, but a measure of the actuation torques when no external forces are applied at the end of the wheel-legs, gives a maximum static friction of about $2 \mathrm{Nm}$ that corresponds, in the worst case to an error of $12 \mathrm{~N}$ at the contact point.

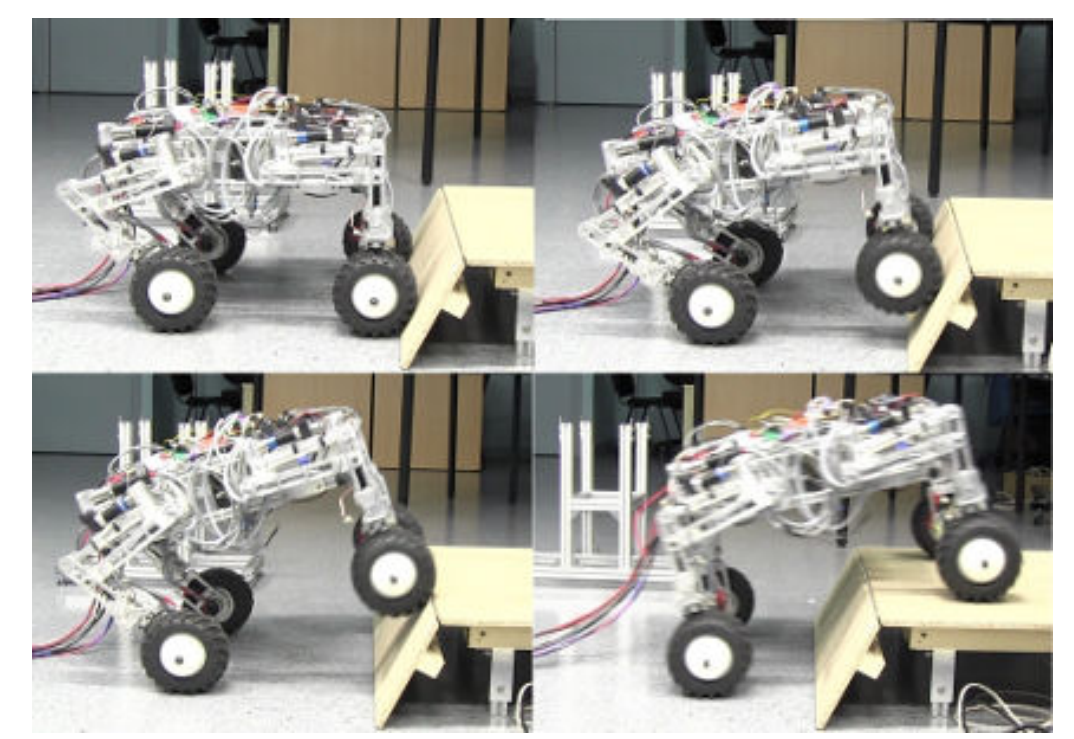

Fig. 6. Evaluation of the control algorithm during step crossing

For sake of clarity, the running timeline is represented by three successive and repetitive phases. During phase 1, the robot wheels are in contact with a flat horizontal ground. Then the front wheels encounter the vertical part of the step and the system enter in phase 2 where adherence conditions at the front wheel become preponderant to insure the robot stability. Thus, we can see that the stability margin drops below zero, indicating that the controller can not produce the necessary forces to start the crossing motion. The desired position of the CoM is calculated and the robot reaches the optimal configuration. During this phase, the robot still uses the internal forces to keep the contact forces inside their friction cones and to sustain its weight. Once the stability margin has increased enough, the robot enters in phase 3 and starts to climb over the step. While climbing, the optimal position of the CoM is updated, avoiding a possible tip-over as the robot is rising. Once the front wheels have reached the 


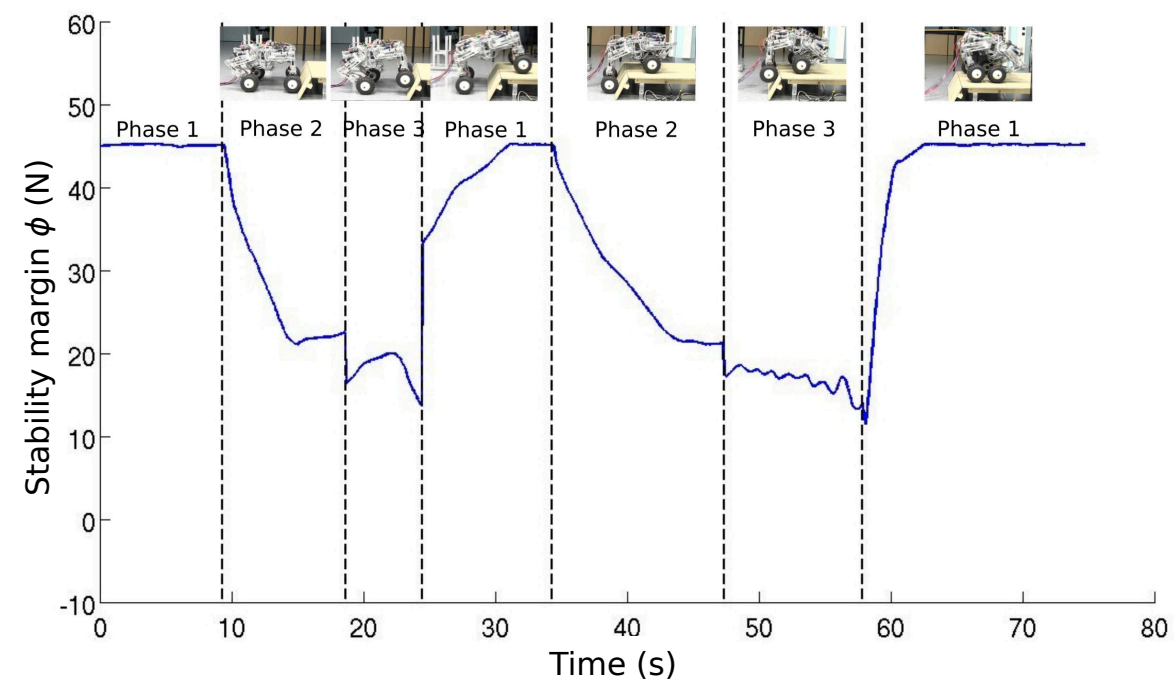

Fig. 7. Stability margin during a step crossing

top of the step, the robot is once again in a phase 1 where all the wheels are on horizontal planes. And, in a similar way, the same phases are following for the rear wheels of the robot.

One can see in Fig. 7 between time $47 s$ to $57 s$ some oscillations during the second "phase 3" that correspond to the crossing of the rear wheels. These perturbations result from the servoing of the CoM position (see Fig. 8) which is perturbed by the impedance control. The main reason is that in this phase some desired joint torques are close to zero which involves some variation of sign around zero and cause the irregular motion of the CoM. Also, we can see in the Fig. 8 that the position of the platform CoM position is quite good excepted again in the phase 3 . This is due to the importance of internal forces during this phase which are critical to insure the obstacle clearance. Thus, the controller try to move the legs in order to control the posture while trying to maintain a large contact forces needed to overcome the low friction at the wheel-ground contacts.

\section{Conclusion}

In this work, we have developed a control algorithm that enhances the climbing performance of a hybrid wheel-legged robot when it crossing a frontal step-like obstacle. The proposed methodology exploits the redundant actuation in order to improve the adhesion at the wheel-ground contacts. This algorithm is based on the optimization of both the robot posture and the distribution of the internal forces. The optimization criterion is based on the measure of the smallest force perturbation sustainable at the contacts level which represents the robustness of the contacts stability in term of traction. Experiments realized on our 


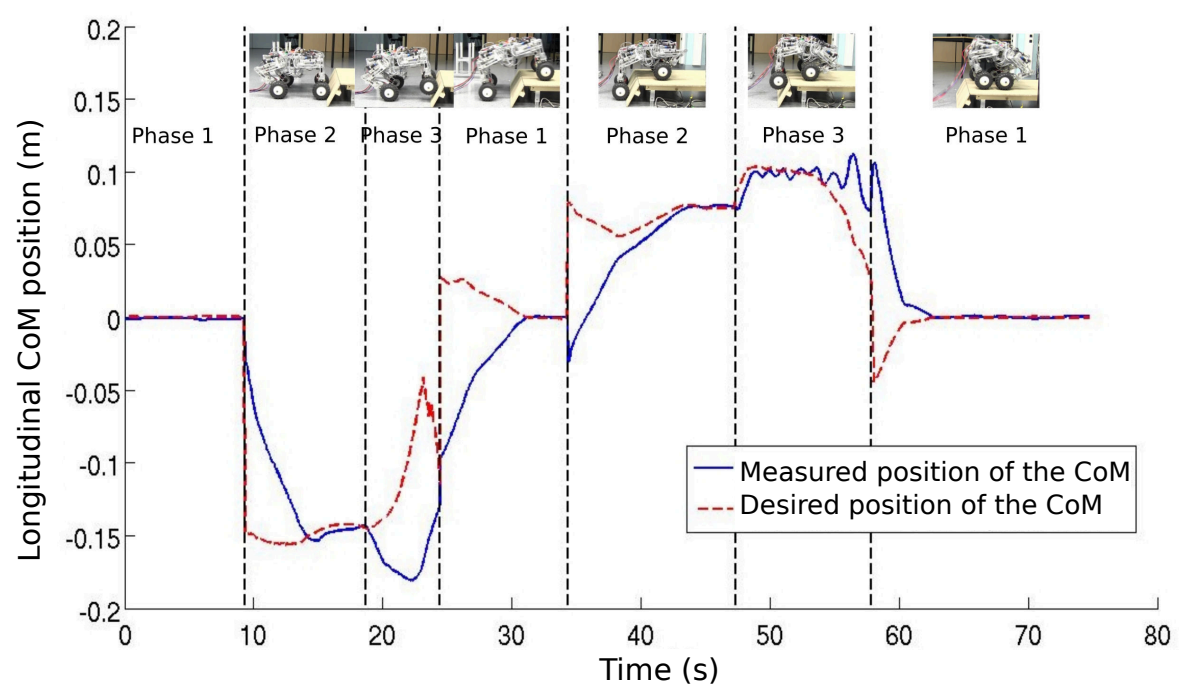

Fig. 8. Desired and actual horizontal position of the CoM

experimental platform Hylos2 validate the implementation of our algorithm on a real system. However, analysis of the posture trajectory shows some discontinuities that should be smoothed. Future works will try to address this problem by including a predictive controller.

\section{References}

1. Aoki, T., Murayama, Y., Hirose, S.: Development of a transformable three-wheeled lunar rover: Tri-star iv. Journal of Field Robotics 31(1), 206-223 (2014)

2. Cheng, F.T., Orin, D.E.: Efficient algorithm for optimal force distribution - the compact-dual lp method. In: IEEE Transaction on Robotics and Automation. vol. 6 (April 1990)

3. Fu, Q., Zhou, X., Krovi, V.: The reconfigurable omnidirectional articulated mobile robot (roamer). In: Khatib, O., Kumar, V., Sukhatme, G. (eds.) Experimental Robotics, Springer Tracts in Advanced Robotics, vol. 79, pp. 871-882. Springer (2014)

4. Grand, C., Ben Amar, F., Plumet, F., Bidaud, P.: Stability control of a wheellegged mini-rover. In: Proc. of CLAWAR'02 : 5th Int. Conf. on Climbing and Walking Robots. pp. 323-330. Paris, France (2002)

5. Grand, C., Amar, F.B., Plumet, F.: Motion kinematics analysis of wheeled-legged robot over $3 \mathrm{~d}$ surface with posture adaptation. Mechanism and Machine Theory 45(3), 477-495 (2010)

6. Hogan, N.: Impedance control: An approach to manipulation. In: American Control Conference, 1984. pp. 304-313. IEEE (1984)

7. Kerr, J., Roth, B.: Analysis of multifingered hands. International Journal of Robotic Research 4, 3-17 (1986) 
8. Quigley, M., Conley, K., Gerkey, B., Faust, J., Foote, T., Leibs, J., Wheeler, R., $\mathrm{Ng}$, A.Y.: Ros: an open-source robot operating system. In: ICRA workshop on open source software (2009)

9. Smith, J., Sharf, I., Trentini, M.: Paw: a hybrid wheeled-leg robot. In: Proc. of IEEE Int. Conf. on Robotics and Automation (ICRA) (2006)

10. Wilcox, B.H., Litwin, T., Biesiadecki, J., Matthews, J., Heverly, M., Morrison, J., Townsend, J., Ahmad, N., Sirota, A., Cooper, B.: Athlete: A cargo handling and manipulation robot for the moon. Journal of Field Robotics 24(5), 421-434 (2007)

11. Ylönen, S., Halme, A.: Further development and testing of the hybrid locomotion of workpartner robot. In: Proc of Int. Conf. on Climbing on Walking Robots (CLAWAR) (2002)

12. Yoshioka, T., Takubo, T., Arai, T., Inoue, K.: Hybrid locomotion of leg-wheel asterisk h. Journal of Robotics and Mechatronics 20(3), 403 (2008) 